FRIEDRICH ENGELS

\title{
ANTEIL DER ARBEIT \\ AN DER MENSCHWERDUNG \\ DES AFFEN
}

DIETZ VERLAG BERLIN 1954 


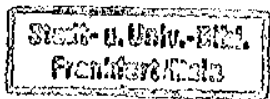

6. Auflage $\cdot 241 .-260$, Tausend

Dictz Verlag GmbH, Berlin + 1, Auflage 1946. Printed in Germany . Alle Rechte vorbelialten - Gestaltung und Typographie: Dictz Entwurf + Papierformat:

$61 \times 86 \mathrm{~cm}$ - Autorenbogen: 1 . Druckbogen: 1,25 . Lizenznummer 1 Druck: Aufjau-Druckerei Käthen
Die ihrem Umfang nach kleine Schrift von Engels über den "Anteil der Arbeit an der Menschwerdung des Affen" ist von gewaltiger wissenschaftlicher Bedeutung. Eine äußerst volkstümliche Darlegungsweise vereinigt sich in ihr mit einer tiefschürfenden Analyse der wichtigsten Probleme, die an der Grenze zwischen der Naturwissenschaft und der Lehre von der mensch- . lichen Gesellschaft liegen.

Die Frage nach dem Ursprung des Menschen gehört zu den interessantesten und bedeutsamsten Problemen der Wissenschaft. Sie ist das Objekt eines besonders erbitterten Kampfes zwischen Wissenschaft und Pfaffentum, Materialismus und Idealismus. Darwin schuf die feste wissenschaftliche Grundlage zur Lösung des Problems auf biologischem Gebiet. Jedoch die andere - die soziologische - Seite der Frage blieb bei Darwin unberücksichtigt. Deshalb vermochten, wie Engels aufzeigt, selbst die am meisten materialistisch eingestellten Naturforscher der Darwinschen Schule sich keine klare Vorstellung von der Entstehung des Menschen zu machen, insofern sic, unter dem Einfluß des Idealismus, die Rolle nicht erkannten, die die Arbeit in diesem Prozeß spielte.

Erst Engels erklärt mit unübertroffener Meisterschaft die hervorragende und ausschlaggebende Rolle der Arbeit bei der Herausbildung des Menschen als physischem Typus und bei der Entstehung der menschlichen Gesellschaft, indem er aufzeigt, wie sich im Laufe der Zeit aus dem Affen eine qualitativ neue Gattung - der Mensch - entwickelt hat. Engels verfolgt die Rolle der Arbeit im Prozeß der Menschwerdung und der nachfolgenden Evolution der menschlichen Gesellschaft, deckt dabei beiläufig die historischen Ursachen auf, die auf einer gewissen Entwicklungsstufe zur Entstehung einer idealistischen Weltanschauung geführt laben, und weist auf die Prozesse hin, die die

2 Engcla, Antell der Arbeit 
„widersinnige und widernatürlicheVorstellung von einem Gegensatz zwischen Geist und Materie, Mensch und Natur, Seele und Leib" nunmehr zu ciner Unmöglichkeit machen.

Engels schrieb den Aufsatz „Anteil der Arbeit an der Menschwerdung.des Affen" ursprünglich als Einleitung zu einer größeren Arbeit "Uber die drei Grundformen der Knechtschaft". Diesen Titel änderte Engels später in „Die Knechtung des Arbeiters". Da jedoch diese Arbeit unvollendet blieb, gab Engels schließlich dem fertig vorliegenden einleitenden Teil, der dem Inhalt der ersten 8 bis 9 Seiten des Manuskripts entspricht, den Titel „Anteil der Arbeit an der Menschwerdung des Affen". (Die letzten 2 bis 3 Seiten des Manuskripts bilden den Übergang zur Behandlung des eigentlichen Themas, der Knechtung der werktätigen Menschheit.) Die Arbeit entstand offenbar im Jahre 1876. Für diese Annahme spricht ein Brief von Wilhelm Liebknecht an Engels vom 10. Juni 1876, in dem Liebknecht unter anderem schreibt: "Die für den ,Volksstaat" versprochene Arbeit, Ưber die drei Grundformen der Knechtschaft" wird mit Schmerzen erwartet." Nach Engels' Tode (1895) wurde das Fragment in seinem literarischen Nachlaß vorgefunden und 1896 von $\mathrm{E}$. Bernstein in der "Nẹen Zeit" veröffentlicht. (Jahrg. XIV, Bd. 2, K. 545-554.)

Der Text der vorliegenden Ausgabe wurde der Neuausgabe des Werkes "Dialektik der Natur" entnommen, die nach der Marx/Engels Gesamtausgabe, Friedrich Engels „Herrn Eugen Dührings Umwälzung der Wissenschaft. Dialektik der Natur", Sonderausgabe Moskau-Leningrad 1935, unter Berücksichtigung der auf Grund eines späteren erneuten Vergleichs mit den Fotokopien des Engelsschen Manuskripts im Marx-Engels-Lenin-Institut, Moskau, erfolgten Korrekturen vorgenommen wurde.

Der Verlag
Die Arbeit ist die Quelle alles Reichtums, sagen die politischen Okonomen. Sie ist dies - neben der Natur, die ihr den Stoff liefert, den sie in Reichtum verwandelt. Aber sie ist noch unendlich mehr als dies. Sie ist die erste Grundbedingung alles menschlichen Lebens, und zwar in einem solchen Grade, daß wir in gewissem Sinn sagen müssen: Sie hat den Menschen selbst geschaffen.

Vor mehreren hunderttausend Jahren, während eines noch nicht fest bestimmbaren Abschnitts jener Erdperiode, die die Geologen die tertiäre nennen, vermutlich gegen deren Ende, lebte irgendwo in der heißen Erdzone - wahrscheinlich auf einem großen, jetzt auf den Grund des Indischen Ozeans versunkenen Festlande - ein Geschlecht menschenähnlicher Affen von besonders hoher Entwicklung. Darwin hat uns eine annähernde Beschreibung dieser unsrer Vorfahren gegeben. Sie waren über und über behaart, hatten Bärte und spitze Ohren, und lebten in Rudeln auf Bäumen.

Wohl zunächst durch ihre Lebensweise veranlaßt, die beim Klettern den Händen andre Geschäfte zuweist als den Füßen, fingen diese Affen an, iuf ebner Erde sich der Beihilfe der Hände beim Gehen zu entwöhnen und einen mehr und mehr aufrechten Gang anzunehmen. Damit war der entscheidende Schritt getan für den Ubergang vom Affen zum Menschen.

Alle noch jetzt lebenden menschenähnlichen Affen können aufrecht stehn und sich auf den beiden Füßen allein fortbewegen. Aber nur zur Not und höchst unbehilflich. Ihr natürlicher Gang geschieht in halb aufgerichteter Stellung und schließt den Gebrauch der Hände ein. Die meisten stützen die Knöchel der Faust auf den Boden und schwingen den Körper mit eingezogenen 
Beinen zwischen den langen Armen durch, wie ein Lahmer, der auf Krücken geht. Uberhaupt können wir bei den Affen alle Ǔbergangsstufen vom Gehen auf allen vieren bis zum Gang auf den beiden Füßen noch jetzt beobachten. Aber bei keinem von ihnen ist der letztere mehr als ein Notbehelf gervorden.

Wenn der aufrechte Gang bei unsern behaarten Vorfahren zuerst Regel und mit der Zeit eine Notwendigkeit werden sollte, so setzt dies voraus, daß den Händen inzwischen mehr und mehr anderweitige Tätigkeiten zufielen. Auch bei den Affen herrscht schon eine gewisse Teilung der Verwendung von Hand und Fuß. Die Hand wird, wie schon erwähnt, beim Klettern in andrex Weise gebraucht als der Fuß. Sie dient vorzugsweise zum Pflücken und Festhalten der Nahrung, wie dies schon bei niederen Säugetieren mit den Vorderpfoten geschieht. Mit ihr bauen sich manche Affen Nester in den Bäumen oder gar, wie der Schimpanse, Dächer zwischen den $Z$ weigen zum Schutz gegen die Witterung. Mit ihr ergreifen sie Knüttel zur Verteidigung gegen Feinde oder bombardieren diese mit Früchten und Steinen. Mit ihr vollziehen sie in der Gefangenschaft eine Anzahl einfacher, den Menschen abgesehener Verrichtungen. Aber grade hier zeigt sich, wie groß der Abstand ist zwischen der unentwickelten Hand selbst der menschenähnlichsten Affen und der durch die Arbeit von Jahrhunderttausenden hoch ausgebildeten Menschenhand. Die ZahI und allgemeine Anordnung der Knochen und Muskel stimmen bei beiden; aber die Hand des niedrigsten Wilden kann Hunderte von Verrichtungen ausführen, die keine Affenhand ihr nachmacht. Keine Affenhand hat je das rohste Steinmesser verfertigt.

Die Verrichtungen, denen unsre Vorfahren im Übergang vom Affen zum Menschen im Lauf vieler Jahrtausende allmählich ihre Hand anpassen lernten, können daher anfangs nur sehr einfache gewesen sein. Die niedrigsten Wilden, selbst diejenigen, bei denen ein Rückfall in einen mehr tierähnlichen Zustand mit gleichzeitiger körperlicher Rückbildung anzunehmen ist, stehn immer noch weit höher als jene Übergangsgeschöpfe. Bis der erste Kiesel durch Menschenhand zum Messer verarbeitet wurde, darüber mögen Zeiträume verflossen sein, gegen die die uns bekannte geschichtliche Zeit unbedeutend erscheint. Aber der entscheidende Schritt war getan: Die Hand war frei geworden und konnte sich nun immer neue Geschicklichkeiten erwerben, und die damit erworbene größere Biegsamkeit vererbte und vermehrte sich von Geschlecht zu Geschlecht.

So ist die Hand nicht nur das Organ der Arbeit, sie ist auch ihr Produkt. Nur durch Arbeit, durch Anpassung an immer neue Verrichtungen, durch Vererbung der dadurch erworbenen besondern Ausbildung der Muskel, Bänder, und in längeren Zeiträumen auch der Knochen, und durch immer erneuerte Anwendung dieser vererbten Verfeinerung auf neue, stets verwickeltere Verrichtungen hat die Menschenhand jenen hohen Grad von Vollkommenheit erhalten, auf dem sie Raffaelsche Gemälde, Thorvaldsensche Statuen, Paganinische Musik hervorzaubern konnte.

Aber die Hand stand nicht allein. Sie war nur ein einzelnes Glied eines ganzen, höchst zusammengesetzten Organismus. Und was der Hand zugute kam, kam auch dem ganzen Körper zugute, in dessen Dienst sie arbeitete - und zwar in doppeiter Weise.

Zuerst infolge des Gesetzes der Korrelation des Wachstums, wie Darwin es genannt hat. Nach diesem Gesetz sind bestimmte Formen einzelner Teile eines organischen Wesens stets an gewisse Formen andrer Teile geknüpft, die scheinbar gar keinen Zusammenhang mit jenen haben. So haben alle Tiere, welche rote Blutzellen ohne Zellenkern besitzen und deren Hinterkopf mit dem ersten Rückgratswirbel durch zwei Gelenkstellen (Kondylen) verbunden ist, ohne Ausnahme auch Milchdrüsen zum Säugen der Jungen. So sind bei Säugetieren gespaltene Klauen regelmäßig mit dem mehrfachen Magen zum Wiederkäuen verbunden. Anderungen bestimmter Formen ziehn Änderungen der Form andrer Körperteile nach sich, ohne daß wir den Zusammen- 
hang erklären können. Ganz weiße Katzen mit blauen Augen sind immer, oder beinahe immer, taub. Dic allmähliche Verfeinerung der Menschenhand und die mit ihr Schritt haltende Ausbildung des Fußes für den aufrechten Gang hat unzweifelhaft auch durch solche Korrelation auf andre Teile des Organismus rückgewirkt. Doch ist diese Einwirkung noch viel zu wenig untersucht, als daß wir hier mehr tun könnten, als sie allgemein konstatieren.

Weit wichtiger ist die direkte, nachweisbare Rückwirkung der - Entwicklung der Hand auf den übrigen Organismus. Wie schon gesagt, waren unsre äffischen Vorfahren gesellig; es ist augenscheinlich unmöglich, den Menschen, das geselligste aller Tiere, von einem ungeselligen nächsten Vorfahren abzuleiten. Die mit der Ausbildung der Hand, mit der Arbeit, beginnende Ferrschaft über die Natur erweiterte bei jedem neuen Fortschritt den Gesichtskreis des Menschen. An den Naturgegenständen entdeckte er fortwährend neue, bisher unbekannte Eigenschaften. Andrerseits trug die Ausbildung der Arbeit notwendig dazu bei, die Gesellschaftsglieder näher aneinanderzuschließen, indem sie die Fälle gegenseitiger Unterstützung, gemeinsamen Zusammenwirkens vermehrte und das Bewußtsein von der Nützlichkeit dieses Zusammenwirkens für jeden einzelnen klärte. Kurz, die werdenden Menschen kamen dahin, daß sie einander etwas zu sagen hatten. Das Bedürfnis schuf sich sein Organ: Der unentwickelte Kehlkopf des Affen bildete sich langsam aber sicher um, durch Modulation für stets gesteigerte Modulation, und die Organe des Mundes lernten allmählich einen artikulierten Buchstaben nach dem andern aussprechen.

Daß diese Erklärung der Entstehung der Sprache aus und mit der Arbeit die einzig richtige ist, beweist der Vergleich mit den Tieren. Das wenige, was diese, selbst die höchstentwickelten, einander mitzuteilen haben, können sie einander auch ohne artikulierte Sprache mitteilen. Im Naturzustand fühlt kein Tier es als einen Mangel, nicht sprechen oder menschliche Sprache nicht verstehn zu können. Ganz anders, wenn es durch Menschen gezähmt ist. Der Hund und das Pferd haben im Umgang mit Menschen cin so gutes $\mathrm{Ohr}$ für artikulierte Sprache erhalten, daß sic jede Sprache leicht soweit verstehn lernen, wie ihr Vorstellungskreis reicht. Sie haben sich ferner die Fähigkeit für Empfindungen wie Anhänglichkeit an Menschen, Dankbarkeit usw. erworben, die ihnen früher fremd waren; und wer viel mit solchen Tieren umgegangen ist, wird sich kaum der Uberzeugung verschließen können, daß es Fälle genug gibt, wo sie jetzt die Unfähigkeit zus sprechen als einen Mangel empfinden, dem allerdings bei ihren allzusehr in bestimmter Richtung spezialisierten Stimmorganen leider nicht mehr abzuhelfen ist. Wo aber das Organ vorhanden ist, da fällt auch diese Unfähigkeit innerhalb gewisser Grenzen weg. Die Mundorgane der Vögel sind sicher so verschieden wie nur möglich von denen des Menschen, und doch sind Vögel die einzigen Tiere, die sprechen lernen; und der Vogel mit der abscheulichsten Stimme, der Papagei, spricht am besten. Man sage nicht, er verstehe nicht, was er spricht. Allerdings wird er aus reinem Vergnügen am Sprechen und an der Gesellschaft von Menschen stundenlang seinen ganzen Wortreichtum plappernd wiederholen. Aber soweit sein Vorstellungskreis reicht, soweit kann or auch verstehen lernen, was er sagt. Man lehre einen Papagei Schimpfwörter, so daß er eine Vorstellung von ihrer Bedeutung bekommt (ein Hauptvergnügen aus heißen Ländern zurücksegelnder Matrosen); man reize ihn, und man wird bald finden, daß er seine Schimpfwörter ebenso richtig zu verwerten weiß wie eine Berliner Gemüsehökerin. Ebenso beim Betteln um Leckereien.

Arbeit zuerst, nach und dann mit ihr die Sprache - das sind die beiden wesentlichsten Antriebe, unter deren Einfluß das Gehirn eines Affen in das bei aller Ahnlichkeit weit größere und vollkommnere eines Menschen allmählich übergegangen ist. Mit der Fortbildung des Gehirns aber ging Hand in Hand die Fortbildung seiner nächsten Werkzeuge, der Sinnesorgane. Wie schon 
die Sprache in ihrer allmählichen Ausbildung notwendig begleitet wird von einer entsprechenden Verfeinerung des Gehörorgarss, so die Ausbildung des Gehirns überhaupt von der der sämtlichen Sinne. Der Adler sieht viel weiter als der Mensch, aber des Menschen Auge sieht viel mehr an den Dingen als das des Adlers. Der Hund hat eine weit feinere Spürnase als der Mensch, aber er unterscheidet nicht den hundertsten Teil der Gerüche, die für diesen bestimmte Merkmale verschiedner Dinge sind. Und der Tastsinn, der beim Affen kaum in seinen rohsten Anfängen existiert, ist erst mit der Menschenhand selbst, durch die Arbeit, herausgebildet worden. -

Die Rückwirkung der Entwidklung des Gehirns und seiner dienstbaren Sinne, des sich mehr und mehr klärenden Bewußtseins, Abstraktions- und Schlußvermögens auf Arbeit und Sprache gab beiden immer neuen Anstoß zurWeiterbildung, einer Weiterbildung, die nicht etwa einen Abschluß fand, sobald der Mensch endgültig vom Affen geschieden war, sondern die seitdem bei verschiednen Völkern und zu verschiednen Zeiten verschieden nach Grad und Richtung, stellenweise selbst unterbrochen durch örtlichen und zeitlichen Rückgang, im ganzen und großen gewaltig vorangegangen ist; einerseits mächtig vorangetrieben, andrerseits in bestimmtere Richtungen gelenkt durch ein mit dem Auftreten des fertigen Menschen neu hinzutretendes Element die Gesellschaft.

Hunderttausende von Jahren - in der Geschichte der Erde nicht mehr als eine Selcunde im Menschenleben ${ }^{1}$ - sind sicher vergangen, che aus dem Rudel baumkletternder Affen eine Gesellschaft von Menschen hervorgegangen war. Aber schließlich war sie da. Und was finden wir wieder als den bezeichnenden

1 Eine Autorität ersten Rangs in dieser Beziehung, Sir W. Thomson, hat berechnet, daß nicht viel mohr als lundert Millionen Jahre verflossen sein können seit der Zcit, wo die Erde soweit abgekühlt war, daß Planzen und Tiere auf ihr leben kounten.
Untcrschied zwischen Affenrudel und Menschengesellschaft? Die Arbeit. Das Affenrudel begnügte sich damit, seinen Futterbezirk abzuweiden, der ihm durch die geographische Lage oder durch den Widerstand benachbarter Rudel zugeteilt war; es unternahm Wanderungen und Kämpfe, um neues Futtergebiet zu gewinnen, aber es war unfähig, aus dem Futterbezirk mehr herauszuschiagen, als er von Natur bot, außer daß es ihn unbewußt mit seinen Abfällen düngte. Sobald alle möglichen Futterbezirke besetzt waren, konnte keine Vermehrung der Affenbevölkerung mehr stattfinden; die Zahl der Tiere konnte sich höchstens gleichbleiben. Aber bei allen Tieren findet Nahrungsverschwendung in hohem Grade statt, und daneben Ertötung des Nahrungsnachwuchses im Keime. Der Wolf schont nicht, wie der Jäger, die Rehgeiß, die ihm im nächsten Jahr die Böcklein liefern soll; die Ziegen in Griechenland, die das junge Gestrüpp abweiden, eh es heranwächst, haben alle Berge des Landes kahlgefressen. Dieser „Raubbau" der Tiere spielt bei der allmählichen Umwandlung der Arten eine wichtige Rolle, indem er sie zwingt, andrer als der gewohnten Nahrung sich anzubequemen, wodurch ihr Blut andre chemische Zusammensetzung bekommt und die ganze Körperkonstitution allmählich eine andre wird, während die einmal fixierten Arten absterben. Es ist nicht zu bezweifeln, daß dieser Raubbau mächtig zur Menschwerdung unsrer Vorfahren beigetragen hat. Bei einer Affenrasse, die an Intelligenz und Anpassungsfähigkeit allen andern weit voraus war, mußte er dahin führen, daß die Zahl der Nahrungspflanzen sich mehr und mehr ausdehnte, daß von den Nahrungspflanzen mehr und mehr eßbare Teile zur Verzehrung kamen, kurz, daß die Nahrung immer mannigfacher wurde und mit ihr die in den Körper eingehenden Stoffe, die chemischen Bedingungen der Menschwerdung. Das alles war aber noch keine eigentliche Arbeit. Die Arbeit fängt an mit der Verfertigung von Werkzeugen. Und was sind die ältesten Werkzeuge, die wir vorfinden? Die ältesten, nach den vorgefundenen Erbstücken vorgeschichtlicher Menschen und nach der Le- 
bensweise der frühesten geschichtlichen Völker wie der rohester jetzigen Wilden zu urteilen? Werkzeuge der Jagd und des Fischfangs, erstere zugleich Waffen. Jagd und Fischfang aber setzen den Übergang von der bloßen Pflanzennahrung zum Mitgenuß des Fleisches voraus, und hier haben wir wieder einen wesentlichen Schritt zur Menschwerdung. Die Fleischkost enthielt in fast fertigem Zustand die wesentlichsten Stoffe, deren der Körper zu seinem Stoffwechsel bedarf; sie kürzte mit der Verdauung die Zeitdauer der übrigen vegetativen, dem Pflanzenleben entsprechenden Vorgänge im Körper $a b$ und gewann damit mehr Zeit, mehr Stoff und mehr Lust für die Betätigung des eigentlich tie-. rischen (animalischen) Lebens. Und je mehr der werdende Mensch sich von der Pflanze entfernte, desto mehr erhob er sich auch über das Tier. Wie die Gewöhnung an Pflanzennahrung neben dem Fleisch die wilden Katzen und Hunde zu Dienern des Menschen gemacht, so hat die Angewöhnung an die Fleischnahrung neben der Pflanzenkost wesentlich dazu beigetragen, dem werdenden Menschen Körperkraft und Selbständigkeit zu geben. Am wesentlichsten aber war die Wirkung der Fleischnahrung auf das Gehirn, dem nun die zu seiner Ernährung und Entwicklung nötigen Stoffe weit reichlicher zuflossen als vorher, und das sich daher von Geschlecht zu Gesdhlecht rascher und vollkommener ausbilden konnte. Mit Verlaub der Herren Vegetarianer, der Mensch ist nicht ohne Fleischnahrung zustande gekommen, und wenn die Fleischnahrung auch bei allen uns bekannten Völkern zu irgendeiner Zeit einmal zur Menschenfresserei geführt hat (die Vorfahren der Berliner, die Weletaben oder Wilzen, aßen ihre Eltera noch im 10. Jahrhundert), so kann uns das heute nichts mehr ausmachen.

Die Fleischkost führte zu zwei neuen Fortschritten von entscheidender Bedeutung: zur Dienstbarmachung des Feuers und zur Zähmung von Tieren. Die erstere kürzte den Verdauungsprozeß noch mehr ab, indem sie die Xost schon sozusagen halbverdaut an den Mund brachte; die zweite machte die Fleischkost reichlicher, indem sie neben der Jagd eine neue regelmäßigere Bezugsquelle dafür eröffnete, und lieferte außerdem in der Milch und ihren Produkten ein neues, dem Fleisch an Stoffmischung mindestens gleichwertiges Nahrungsmittel. So wurden beide schon direkt neue Emanzipationsmittel für den Menschen; auf ihre indirekten Wirkungen im einzelnen einzugehn, würde uns hier zu weit führen, von so hoher Wichtigkeit sie auch für die Entwicklung des Mensdien und der Gesellschaft gewesen sind.

Wie der Mensch alles Eßbare essen lernte, so lernte er auch in jedem Klima leben. Er verbreitete sich über die ganze bewohnbare Erde, er, das einzige Tier, das in sich selbst die Machtvollkommenheit dazu besaß. Die andren Tiere, die sich an alle Klimata gewöhnt haben, haben dies nicht aus sich selbst, nur im $\mathrm{Ge}$ folge des Menschen, gelernt: Haustiere und Ungeziefer. Und der Ubergang aus dem gleichmäßig heißen Klima der Urheimat in kältere Gegenden, wo das Jahr sich in Winter und Sommer teilte, schuf neue Bedürfnisse: Wohnung und Kleidung zum Schutz gegen Kälte und Nässe, neue Arbeitsgebiete und damit neue Betätigungen, die den Menschen immer weiter vom Tier entfernten.

Durch das Zusammenwirken von Hand, Sprachorganen und Gehirn nicht allein bei jedem einzelnen, sondern auch in der Gesellschaft, wurden die Menschen befähigt, immer verwickeltere Verrichtungen auszuführen, immer höhere Ziele sich zu stellen und zu erreichen. Die Arbeit selbst wurde von Geschlecht zu Geschlecht eine andre, vollkommnere, vielseitigere. Zur Jagd und Viehzucht trat der Ackerbau, zu diesem Spinnen und Weben, Verarbeitung der Metalle, Töpferei, Schiffahrt. Neben Handel und Gewerbe trat endlich Kunst und Wissenschaft, aus Stämmen wurden Nationen und Staaten. Recht und Politik entwickelten sich, und mit ihren das phantastische Spiegelbild der menschlichen Dinge im menschlichen Kopf: dic Religion. Vor allen diesen Gebilden, die zunächst als Produkte des Kopfs sich darstellten und die die menschlichen Gesellschaften zu beherrschen schienen, traten die bescheidneren Exzeugnisse der arbeitenden 
Hand in den Hintergrund; und zwar um so mehr, als der die Arbeit planende Kopf schon auf einer sehr frühen Entwicklungsstufe der Gesellschaft ( $z$. B. schon in der einfachen Familie) die geplante Arbeit durch andre Hände ausführen lassen konnte als die seinigen. Dem Kopf, der Entwicklung und Tätigkeit des Gehirns, wurde alles Verdienst an der rasch fortschreitenden Zivilisation zugeschrieben; die Menschen gewöhnten sich daran, ihr Tun aus ihrem Denken zu erklären statt aus ihren Bedürfnissen (die dabei allerdings im Kopf 'sich widerspiegeln, zum Bewußtsein kommen) - und so entstand mit der Zeit jene idealistische Weltanschauung, die namentlich seit Untergang der antiken Welt die Köpfe beherrscht hat. Sie herrscht noch so sehr, daß selbst die materialistischsten Naturforscher der Darwinschen Schule sich noch keine klare Vorstellung von der Entstehung des Menschen machen können, weil sie unter jenem ideologischen Einfluß die Rolle nicht erkennen, die die Arbeit dabei gespielt hat.

"Die Tiere, wie schon angedeutet, verändern durch ihre Tätigkeit die äußere Natur ebenșogut, wenn auch nicht in dem Maße wie der Mensch, und diese durch sie vollzogenen Änderungen ihrer Umgebung wirken, wie wir sahen, wieder verändernd auf ihre Urheber zurück. Denn in der Natur geschieht nichts vercinzelt. Jedes wirkt aufs andre und umgekehrt, und es ist meist das Vergessen dieser allseitigen Bewegung und Wechselwirkung, das unsre Naturforscher verhindert, in den einfachsten Dingen klar zu sehn. Wir sahen, wie die Ziegen die Wiederbewaldung von Griechenland verhindern; in Sankt Helena haben die von den ersten Anseglern ans Land gesetzten Ziegen und Schweine es fertiggebracht, die alte Vegetation der Insel fast ganz auszurotten, und so den Boden bereitet, auf dem die von spätern Schiffern und Kolonisten zugeführten Pflanzen sich ausbreiten konnten. Aber wenn die Tiere eine dauernde Einwirkung auf ihre Umgebung ausüben, so geschieht dies unabsichtlich, und ist, für diese Tiere selbst, etwas Zufälliges. Je mehr dic Menschen sich aber vom Tier entfernen, desto mehr nimmt ihre Einwirkung auf die Natur den Charakter vorbedachter, planmäßiger, auf bestimmte, vorher belcannte Ziele gerichteter Handlung an. Das Tier vernichtet die Vegetation eines Landstrichs, ohne zu wissen, was es tut. Der Mensch vernichtet sie, um in den freigewordnen Boden Feldfrüchte zu säen oder Bäume und Reben zu pflanzen, von denen er weiß, daß sie ihm ein Vielfaches der Aussaat einbringen werden. Er versetzt Nutzpflanzen und Haustiere von einem Land ins andre, und ändert so die Vegetation und das Tierleben ganzer Weltteile. Noch mehr. Durch künstliche Züchtung werden Pflanzen wie Tiere unter der Hand des Menschen in einer Weise verändert, daß sie nicht wiederzuerkennen sind. Die wilden Pflanzen, von denen unsre Getreidearten abstammen, werden noch vergebens gesucht. Von welchem wilden Tier unsre Hunde, die selbst unter sich so verschieden sind, oder unsre ebenso zahlreichen Pferderassen abstammen, ist noch immer streitig.

Es versteht sich übrigens von selbst, daß es uns nicht einfällt, den Tieren die Fähigkeit planmäßiger, vorbedachter Handlungsweise abzustreiten. Im Gegenteil. Planmäßige Handlungsweise existiert im Keime schon überall, wo Protoplasma, lebendiges Eiweiß existiert und reagiert, d. h. bestimmte, wenn auch noch so einfache Bewegungen als Folge bestimmter Reize von außen vollzieht. Solche Reaktion findet statt, wo noch gar keine Zelle, geschweige eine Nervenzelle, besteht. Die Art, wie insektenfressende Pflanzen ihre Beute abfangen, erscheint ebenfalls in gewisser Beziehung als planmäßig, obwohl vollständig bewußtlos. Bei den Tieren entwickelt sich die Fähigkeit bewußter, planmäßiger Aktion im Verhältnis zur Entwicklung des Nervensystemș und erreicht bei den Säugetieren eine schon hohe Stufe. Auf der englischen Fuchsparforcejagd kann man täglich beobachten, wie genau der Fuchs seine große Ortskenntnis zu verwenden weiß, um seinen Verfolgern zu entgehn, und wie gut er alle Bodenvorteile kennt und benutzt, die die Fährte unterbrechen. Bei unsern im Umgang mit Menschen höher entwickelten Haustieren kann man tagtäglich Streiche der Schlauheit beobachten, die 
mit denen menschlicher Kinder ganz auf derselben Stufe stehn. Denn wie die Entwicklungsgeschichte des menschlichen Keims im Mutterleibe nur eine abgekürzte Wiederholung der millionenjährigen körperlichen Entwicklungsgeschichte unsrer tierischen Vorfahren, vom Wurm angefangen, darstellt, so die geistige Entwicklung des menschlichen Kindes eine, nur noch mehr abgekürzte, Wiederholung der intellektuellen Entwicklung derselbea Vorfahren, wenigstens der späteren. Aber alle planmäßige Aktion aller. Tiere hat es nicht fertiggebracht, der Erde den Stempel ihres Willens aufzudrücken. Dazu gehörte der Mensch.

Kurz, das Tier benutzt die äußere Natur bloß und bringt Änderungen in ihr einfach durch seine Anweserheit zustande; der Mensch macht sie durch seine Anderungen seinen Zwecken dienstbar, beherrscht sie. Und das ist der letzte, wesentliche Unterschied des Menschen von den übrigen Tieren, und es ist wieder die Arbeit, die diesen Unterschied bewirkt ${ }^{1}$.

Schmeicheln wir uns indes nicht zu sehr mit unsern menschlichen Siegen über die Natur. Für jeden solchen Sieg rächt sie sich an uns. Jeder hat in erster Linie zwar dic Folgen, auf die wir gerechnet, aber in zweiter und dritter Linie hat er ganz andre, unvorhergesehene Wirkungen, die nur zu oft jene ersten Folgen wieder aufheben. Die Leute, die in Mesopotamien, Griechenland, Kleinasien und anderswo die Wälder ausrotteten, um urbares Land zu gewinnen, träumten nicht, daß sie damit den Grund zur jetzigen Verödung jener Länder legten, indem sic ihnen mit den Wäldern die Ansammlungszentren und Behälter der Feuchtigkeit entzogen. Die Italiener der Alpen, als sie die am Nordabhang des Gebirgs so sorgsam gehegten Tannenwälder am Südabhang vernutzten, ahnten nicht, daß sie damit der Sennwirtschaft auf ihrem Gebiet die Wurzel abgruben; sie ahnten noch weniger, daß sie dadurch ihren Bergquellen für den größten Teil des Jahrs das Wasser entzogen, damit diese

" Am Rande des Ms. ist mit Bleistift vermerkt: "Veredlung". Die Red. zur Regenzeit um so wütendere Flutströme über die Ebene ergießen könnten. Die Verbreiter der Kartoffel in Europa wußten nicht, daß sie mit den mehligen Knollen zugleich die Skrofelkrankheit verbreiteten. Und so werden wir bei jedem Schritt daran erinnert, daß wir keineswegs die Natur beherrschen, wie ein Eroberer ein fremdes Volk beherrscht, wie jemand, der außer der Natur steht - sondern daß wir mit Fleisch und Blut und Hirn ihr angehören und mitten in ihr stehn, und daß unsre ganze Herrschaft über sie darin besteht, im Vorzug vor allen andern Geschöpfen ihre Gesetze erkennen und richtig anwenden zu können.

Und in der Tat lernen wir mit jedem Tage ihre Gesetze richtiger verstehn und die näheren und entfernteren Nachwirkungen unsrer Eingriffe in den herkömmlichen Gang der Natur erkennen. Namentlich seit den gewaltigen Fortschritten der Naturwissenschaft in diesem Jahrhundert werden wir mehr und mehr in den Stand gesetzt, auch die entfernteren natürlichen Nachwirkungen wenigstens unsrer gewöhnlichsten Produktionshandlungen kennen und damit beherrschen zu lernen. Je mehr dies aber geschieht, desto mehr werden sich die Menschen wieder als eins mit der Natur nicht nur fühlen, sondern auch wissen, und je unmöglicher wird jene widersinnige und widernatürliche Vorstellung von einem Gegensatz zwischen Geist und Materie, Mensch und Natur, Seele und Leib, wie sie seit dem Verfall des klassischen Altertums in Europa aufgekommen und im Christentum ihre höchste Ausbildung erhalten hat.

Hat es aber schon die Arbeit von Jahrtausenden erfordert, bis wir einigermaßen lernten, die entferntern natiirlichen Wirkungen unsrer auf die Produktion gerichteten Handlungen zu berechnen, so war dies noch weit schwieriger in bezug auf die entfernteren gesellschaftlichen Wirkungen dieser Handlungen. Wir erwähnten die Kartoffel und in ihrem Gefolge die Ausbreitung der Skrofeln. Aber was sind die Skrofeln gegen die Wirkungen, die die Reduktion der Arbeiter auf Kartoffelnahrung 
auf die Lebenslage der Volksmassen ganzer Länder hatte, gegen die Hungersnot, die 1847 im Gefolge der Kartoffelkrankheit Irland betraf, eine Million kartoffel- und fast nur kartoffelessender Irländer unter die Erde und zwei Millionen über das Meer warf? Als die Araber den Alkohol destillieren lernten, ließen sie sich nicht im Traum einfallen, daß sie damit eins der Hauptwerkzeuge geschaffen, womit die Ureinwohner des damals noch gar nicht entdeckten Amerikas aus der Welt geschafft werden sollten. Und als dann Kolumbus dies Amerika entdeckte, wußte er nicht, daß er damit die in Europa längst überwundne Sklaverei zu neuem Leben erweckte und die Grundlage zum Negerhandel legte. Die Männer, die im siebzehnten und achtzehnten Jahrhundert an der Herstellung der Dampfmaschine arbeiteten, ahnten nicht, daß sie das Werkzeug fertigsteliten, das mehr als jedes andre die Gesellschaftszustände der ganzen Welt revolutionieren und namentlich in Europa durch Konzentrierung des Reichtums auf Seite der Minderzahl, und der Besitzlosigkeit auf Seite der ungeheuren Mehrzahl, zuerst der Bourgeoisie die soziale und politische Herrschaft verschaffen, dann aber einen Klassenkampf zwischen Bourgeoisie und Proletariat erzeugen sollte, der nur mit dem Sturz der Bourgeoisie und der Abschaffung aller Klassengegensätze endigen kann. - Aber auch auf diesem Gebiet lernen wir allmählich, durch lange, oft harte Erfahrung und durch $\mathrm{Zu}-$ sammenstellung und Untersuchung des geschichtlichen Stoffs, uns über die mittelbaren, entfernteren gesellschaftlichen Wirkungen unsrer produktiven Tätigkeit Klarheit zu verschaffen, und damit wird uns die Möglichkeit gegeben, auch diese Wirkungen zu beherrschen und zu regeln.

Um diese Regelung aber durchzuführen, dazu gehört mehr als die bloße Erkenntnis. Dazu gehört eine vollständige Umwälzung unsrer bisherigen Produktionsweise und mit ihr unsrer jetzigen gesamten gesellschaftlichen Ordnung.

Alle bisherigen Produktionsweisen sind nur auf Exziclung des nächsten, unmittelbarsten Nutzeffekts der Arbeit ausgegangen.
Die weiteren erst in späterer Zeit eintretenden, durch allmähliche Wiederholung und Anhäufung wirksam werdenden Folgen blieben gänzlich vernachlässigt. Das ursprüngliche gemeinsame Eigen. tum am Boden entsprach einerseits einem Entwicklungszustand der Menschen, der ihren Gesichtskreis überhaupt auf das Allernächste beschränkte, und setzte andrerseits einen gewissen Ưberfluß an verfügbarem Boden voraus, der gegenüber den etwaigen schlimmen Folgen dieser waldursprünglichen Wirtschaft einen gewissen Spielraum ließ. Wurde dieser Uberschuß von Land erschöpft, so verfiel auch das Gemeineigentum. Alle höheren Formen der Produktion aber sind zur Trennung der Bevölkerung in verschiedne Klassen und damit zum Gegensatz von herrschenden und unterdrückten Klassen vorangegangen; damit aber wurde das Interesse der herrschenden Klasse das treibende Element der Produktion, soweit diese sich nicht auf den notdürftigsten Lebenswititerhalt der Unterdrückten beschränkte. Am vollständigsten ist dies in der jetzt in Westeuropa herrschenden kapitalistischen Produktionsweise durchgeführt. Die einzelnen, Produktion und Austausch beherrschenden Kapitalisten können sich nur um den unmittelbarsten Nutzeffekt ihrer Handlungen kümmern. Ja selbst dieser Nutzeffekt - soweit es sich um den Nutzen des erzeugten oder ausgetauschten Artikels handelt - tritt vollständig in den Hintergrund; der beim Verkauf zu erzielende Profit wird die einzige Triebfeder.

Die Sozialwissenschaft der Bourgeoisie, die klassische politische Okonomie, beschäftigt sich vorwiegend nur mit den unmittelbar beabsichtigten gesellschaftlichen Wirkungen der auf Produktion und Austausch gerichteten menschlichen Handlungen. Dies entspricht ganz der gesellschaftlichen Organisation, deren theoretischer Ausdruck sie ist. Wo einzelne Kapitalisten um des unmittelbaren Profits willen produzieren und austauschen, können in erster Linie nur die nächsten, unmittelbarsten Resultate in Betracht kommen. Wenn der einzelne Fabrikant oder Kaufmann 
die fabrizierte oder eingekaufte "Ware nur mit dem üblichen Profitchen verkauft, so ist er zufrieden, und es kümmert ilın nicht, was nachher aus der Ware und deren Käufer wird. Ebeniso mit den natürlichen Wirkungen derselben Handlungen. Die spanischen Pflanzer in Kuba, die die Wälder an den Abhängen niederbrannten und in der Asche Dünger genug für eine Generation höchst rentabler Kaffeebäume vorfanden - was lag ihnen daran, daß nachher die tropischen Regengüsse die nun schutzlose Dammerde herabschwemmten und nur nackten Fels hinterließen? Gegenüber der Natur, wie der Gesellschaft, kommt bei der heutigen Produktionsweise vorwiegend nur der erste, handgreiflichste Erfolg in Betracht; und dann wundert man sich noch, daß dic entfernteren Nachwirkungen der hierauf gerichteten Handlungen ganz andre, meist ganz entgegengesetzte sind, daß die Harmonie von Nachfrage und Angebot in deren polaren Gegensatz umschlägt, wie der Verlauf jedes zehnjährigen industrielle.. Zyklus ihn vorführt und wie auch Deutschlard im "Krach"1/ ein kleines Vorspiel davon erlebt hat; daß das auf eigne Arbeit gegründete Privateigentum sich mit Notwendigkeit fortentwickelt zur Eigentumslosigkeit der Arbeiter, während aller Besitz sich mehr und mehr in den Händen von Nichtarbeitern konzentriert; $\mathrm{daß}[\ldots]^{2}$.

1 Engels meint die Wirtochaftskrise von 1873/74. Die Red.

2 [...] Flicr bricht das Manuskript ab. Die Red,

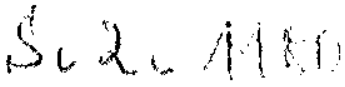

BUCH-NR. 51.098 .269 